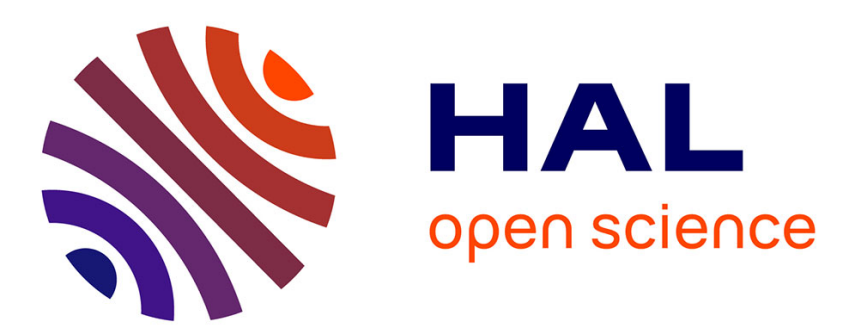

\title{
Catalytic Tubular Microjet Propulsion Model for Endovascular Navigation
}

Bruno Sarkis, David Folio, Antoine Ferreira

\section{To cite this version:}

Bruno Sarkis, David Folio, Antoine Ferreira. Catalytic Tubular Microjet Propulsion Model for Endovascular Navigation. IEEE International Conference on Robotics and Automation (ICRA'2015), IEEE, May 2015, Seattle, Washington, United States. hal-01160826

\section{HAL Id: hal-01160826 \\ https://hal.science/hal-01160826}

Submitted on 8 Jun 2015

HAL is a multi-disciplinary open access archive for the deposit and dissemination of scientific research documents, whether they are published or not. The documents may come from teaching and research institutions in France or abroad, or from public or private research centers.
L'archive ouverte pluridisciplinaire HAL, est destinée au dépôt et à la diffusion de documents scientifiques de niveau recherche, publiés ou non, émanant des établissements d'enseignement et de recherche français ou étrangers, des laboratoires publics ou privés. 


\title{
Catalytic Tubular Microjet Propulsion Model for Endovascular Navigation
}

\author{
Bruno Sarkis $^{1}$, David Folio ${ }^{2}$ and Antoine Ferreira ${ }^{2}$
}

\begin{abstract}
This paper describes the propulsion of the catalytic tubular microjet using Navier-Stokes equations. Especially, the thrust capability of the microjet is outstanding compared with the other microsystems, but remains only partially understood. Studies have identified the internal precursory mechanisms of the propulsion of the microjet: its inner wall catalyzes the dismutation of aqueous hydrogen peroxide, and an oxygen bubble is then formed and migrates towards its widest opening. This impulses the propulsion of the microjet towards the opposite direction. However, the precise propellant role of the liquid surrounding the jet remains misunderstood. The same goes for the inner wall of the vessel in which the jet navigates, especially in narrowed environment. This article discusses these aspects in a simplified theoretical framework. Calculations are performed by explicit computation of NavierStokes equations. The obtained theoretical outcome are in good agreement with experimental results in the literature.
\end{abstract}

\section{INTRODUCTION}

Self-propelled microrobots are an important step towards the design of autonomous microsystems for micromanipulation or biomedical applications [1], [2]. Recently the catalytic microjets have been investigated [2]-[4]. Microjets are microscopic metal rockets in the shape of quasicylindrical hollow truncated cones. They are part of the tube-like microswimmers family, that include several forms of propulsion, such as bubbled, electrophoretic, electric, magnetic and acoustic ones [2]. Catalytic tubular microjets are produced by rolling up a superposition of metal sheets, one of which is the catalyst (see [4]-[7] for the details of their manufacturing techniques). They draw their fuel, including hydrogen peroxide $\left(\mathrm{H}_{2} \mathrm{O}_{2}\right)$, strong acids and hydrazine, from the surrounding liquid (e.g. water or blood medium) and convert it into a bubble of gas by chemical catalysis [7]. The internal surface metal (e.g. platinum: Pt) is the catalyst of this reaction. Thus, their propulsion is ensured with no external energy supply, contrary to the other modes of propulsion, such as magnetic or acoustic ones. In addition to their efficient propulsion, the orientation of the direction of the microjets trajectory is relatively safer compared to other microsystems. For instance, unlike Janus spherical particles, they exhibit a lower natural deflection [8]. Moreover, the catalytic microjet can be magnetically guided [5], [9]. In [5] the contact with living tissues is studied. Therefore, catalytic tubular microjets are prime candidates for the propulsion of more massive microsystems. They are

\footnotetext{
${ }^{1} \mathrm{~B}$. Sarkis is an engineer with the city of Paris, France

${ }^{2}$ D. Folio and A. Ferreira are with INSA Centre Val de Loire, Université d'Orléans, PRISME EA 4229, Bourges, France. Corresponding author: David FOLIO (Email: david.foliodinsa-cvl.fr)
}

one of the most promising ways to propel future medical microrobots, delivery of cargo and many applications [6].

To achieve these goals, it is important to understand the propulsion mechanism of the catalytic microjet. Previous studies [2], [6], [7], [10] have initiated the description of the behavior of the microjet. In particular, its driving motion is different to similar systems such as nanorods. Whereas for nanorods the propulsion is generated from the surface tension created by catalytic reaction [11], the microjet is essentially propelled via a bubble created by catalysis. In [7] the authors have identified the jet-internal phenomenon that induces the propulsion of the microjet: i) creation of a bubble in the head of the jet, ii) migration of this bubble through this tube, and iii) its ejection by the largest orifice at the rear of the jet. This work also proposed to model and to quantify the propulsion of the jet, via the partial transfer of quantity of momentum between the microjet and the moving liquid at both orifices.

Nevertheless, it is necessary to properly model and control the microjet propulsion to ensure the proper execution of future missions. In particular, this includes the study of the influence of the geometry and environment of the microjet. For instance, the dependence of the performance of the jet with respect to its own geometry is presented in [10]. In [6], [10] the authors have studied the influence of the chemical environment on its performance, including the quasi-linear influence of the concentration of aqueous $\mathrm{H}_{2} \mathrm{O}_{2}$ on the velocity of catalytic microjets which have Pt inner surface. These works show that the microjet can reach speeds of around $1 \mathrm{~mm} / \mathrm{s}$, which is well above the speed of $100 \mu \mathrm{m} / \mathrm{s}$ raised in [7]. In [6] the authors warn against chemical catalysis brakes. The geometry of its environment (including the radius of the blood vessel) also influences the performance of the microjet, which remains to be studied.

To improve the understanding of the propulsion of the catalytic microjet, it is still necessary to consider the following three points. First, the motion of the surrounding liquid has to be related to the thrust mechanism. This allows its systemic study and integration to the understanding of the movements of groups of microjets and the interaction with any other object in the surrounding environment, such as blood cells, bacteria, and so on. Secondly, the various aqueous force fields which help propel the microjet have to be identified and distinguished through Navier-Stokes (N.-S.) equations, for the future control of its motion. Thirdly, it should be understood how the geometry of surrounding environment (here blood vessels) influences the microjet propulsion. This allows selecting the most relevant biomedical application framework, and compensating any physicochemical barrier 
to the propulsion. This article aims to address briefly these issues through a qualitative analysis by simplified analytical calculation of the N.-S. equation, in the case of a cylindrical microjet navigating on the centerline of a slender cylinder filled with liquid. Especially, this study will focus on the use of catalytic microjet for future biomedical application through the cardiovascular system [12]. The proposed analytical framework provides a simple understanding of how the "jet-liquid-wall" system parameters help and influence the propulsion of the microjet.

This paper is organized as follows. After describing the context of our study in Section II, Section III presents a modeling of the microjet. The influence of the radius of the vessel on the geometry of flow (and thus the velocity gradients, pressures, shear and microjet propulsion), is studied in Section IV. Finally, Section V discusses the results and some open issues. This study is concluded in Section VI.

\section{BACKGROUNDS}

Among proposed microsystems, catalytic tubular microjets are a promising microtool for future biomedical applications, as they are self-propelled and do not require any supply of energy except for their guidance [1], [2], [13]. Particularly, it is established that such microsystem can be used in innovative minimally invasive surgical procedures [2], [12]. Hence, when the microjets are propelled in the blood circulatory system, a very large number of remote locations in the human body become accessible. However, the human vascular network hemodynamics changes with the vessel radius [12]: from arteries with a large radius of about $R=10 \mathrm{~mm}$, a viscosity of $\eta=3 \mathrm{mPas}$ and a flow velocity of $v_{f}=400 \mathrm{~mm} / \mathrm{s}$; to capillaries with small radius of $R=10 \mu \mathrm{m}$, a viscosity of $\eta=6.5 \mathrm{mPas}$ and a flow less than $v_{f} \leq 1 \mathrm{~mm} / \mathrm{s}$ (see Fig. 1). Thus, it is still challenging driving suitably such wireless microdevices in the human cardiovascular system [12], [14]. Improving the understanding of the interaction of the microsystem in a vascular environment is an important issue.

The basic propulsion principle of a catalytic microjet is as follows: gas is produced by catalysis, and rapidly forms one (or several) bubble(s). This bubble migrates towards the widest opening of the jet, and propels it towards the opposite direction. Fig. 1 illustrates a hydrogen peroxide $\left(\mathrm{H}_{2} \mathrm{O}_{2}\right)$ fueled catalytic tubular microjet in blood vessels. If the phenomenon is qualitatively identified, to date we do not know the quantitative influence of the vessel geometry on the microjet motion. To understand this impact, it is necessary to analyze the jet-bubble-liquid-wall interactions. The flow generates both the propulsion and the brakes of the microjet by its pressure on the bubble and through the shear stress exerted on its inner and outer walls. To quantify these motor and brake, the flow around the microjet should be first characterized. In the reference frame of the microjet which navigates at speed $v_{\text {jet }}$, Fig. 2 represents the flow around the microjet as the superposition of two components: i) the driving flow, due to the migration of the bubble at speed $v_{\mathrm{b}}$ towards the rear of the microjet; and ii) the drag flow,

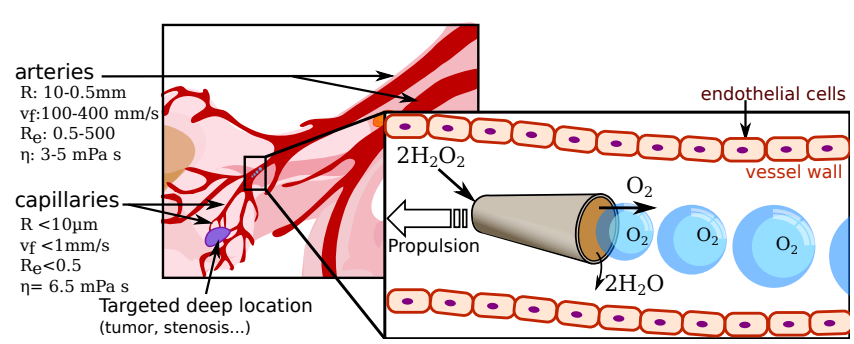

Fig. 1. Schematic representation of (left) the vascular network and (right) the motion of a microjet with hydrogen peroxide in a blood vessel.

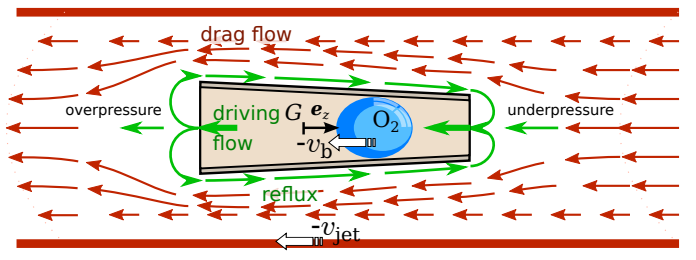

Fig. 2. Flow structure around a microjet, viewed in its reference frame. Flow is the superposition of two components: (green) the migration of the bubble propelling the microjet; (red) the consequence of the motion of the microjet that tends to drag it. Relatively to the microjet, the blood vesse moves at speed $-v_{\mathrm{jet}}$, and the bubble moves at speed $-v_{\mathrm{b}}$.

caused by the recoil velocity of the vessel relatively to the microjet. Due to the migration of the bubble, the driving flow induces an overpressure at the rear of the microjet. In addition, the incompressibility of the flow implies an external backflow around the jet outer wall. These two phenomena tend to propel the microjet. Nevertheless, an induced internal wall shear stress occurs and leads to a brake that pulls back the microjet. Their superposition and impact on the microjet (force and speed) are modeled in the following sections.

\section{MICROJET MODELING}

\section{A. The Microjet and its Bubbles}

Let the parameters $r_{\min }, r_{\max }$ and $l$ resp. denote the minimum and maximum radius, and the length of the microjet, as depicted in Fig. 3. The radii, of few micrometers, are close to each other, and the length is a few tens of micrometers. This geometry ensures a low conical angle, in the order of $\varphi \sim 0.01 \mathrm{rad}[7]$.

The microjet, in its own reference frame, is oriented along a $\mathbf{G z}$-axis, where $G$ is the middle of the segment $A P$ (cf. Fig. 3). At $t=0$ a bubble forms at a random position within the jet, probably closer to the front $A$ than the rear $P$. This bubble becomes tangent to the jet at $t_{0}>0$ and begins to migrate to $P$. The migration speed of the bubble mainly

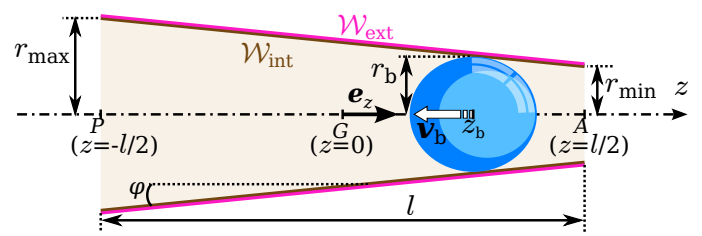

Fig. 3. Schematic representation of a microjet and its bubble. 


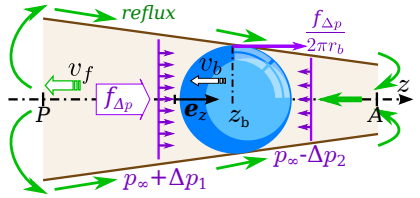

(a)

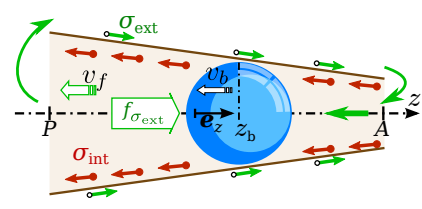

(b)
Fig. 4. The two driving forces of the microjet, from those generated by the flow around the jet, due to the migration of a bubble: (a) pressure force; and (b) shear forces.

depends on its growth rate and on the geometry of the jet. Let $z_{b}$ denote the abscissa of the center of gravity of the bubble, and $\mathbf{v}_{b}=-v_{\mathrm{b}} \cdot \mathbf{e}_{z}$ its speed, where $\mathbf{e}_{z}$ is the unit vector of the $\mathbf{G z}$-axis and $v_{\mathrm{b}} \equiv\left\|\mathbf{v}_{b}\right\|$. Finally, the bubble is ejected at $t_{1} \gg t_{0}$ at $P$ with the velocity $v_{\mathrm{b}}=1.2 \mathrm{~mm} / \mathrm{s}$ [7].

When the bubble reaches the inner wall $\mathcal{W}_{\text {int }}$, the microjet is a microswimmer, and thus has their usual properties. In particular, the distance traveled during a stroke, here the migration of the bubble, is independent of its run time. This suggests a relationship of the type:

$$
v_{\text {jet }} \propto v_{\mathrm{b}}
$$

\section{B. Qualitative Assessment of Forces at Equilibrium}

By migrating, the bubble pushes the liquid situated at the rear $P$ of the microjet, and swallows the flow at the front $A$. It creates a pressure differential between $A$ and $P$ : the pressure at the rear $P$ is $p_{\infty}+\Delta p_{1}$, whereas the pressure at the front $A$ is $p_{\infty}-\Delta p_{2}$ (e.g. $\Delta p_{1}>0$ and $\Delta p_{2}>0$ ). Thus, the difference established, $\Delta p=\Delta p_{1}+\Delta p_{2}$, is the first motor $\mathbf{f}_{\Delta p}$ of the jet, and is depicted in Fig. 4(a). The pressure differential can also be written as follows:

$$
\Delta p=\Delta p_{\text {int }}+\Delta p_{\text {ext }}
$$

where $\Delta p_{\text {int }}$ and $\Delta p_{\text {ext }}$ are formed resp. inside and outside of the microjet. The external component $\Delta p_{\text {ext }}$ can be decomposed as:

$$
\Delta p_{\text {ext }}=\Delta p_{\text {ext, rect. }}+\Delta p_{\text {ext,curv }}
$$

where $\Delta p_{\text {ext, rect. }}$ and $\Delta p_{\text {ext,curv. are components defined }}$ resp. at the level, and upstream/downstream of the jet. From these pressure components result resp. the $\mathbf{f}_{\Delta p_{\text {int }}}, \mathbf{f}_{\Delta p_{\text {ext }}}$, $\mathbf{f}_{\Delta p_{\text {ext, rect. }}}$ and $\mathbf{f}_{\Delta p_{\text {ext, curv. }} \text {. forces. Moreover, the backflow }}$ caused by the external flow creates a shear stress $\sigma_{\text {ext }}$ which also contributes to the microjet propulsion, and is illustrated in Fig. 4(b). The resulting force $\mathbf{f}_{\sigma_{\text {ext }}}$ is then the second jet's engine. Furthermore, due to the conservation law the internal engine $\mathbf{f}_{\Delta p_{\text {int }}}$ is compensated by the internal shear stress force $\mathbf{f}_{\sigma_{\text {int }}}$, that is: $\mathbf{f}_{\Delta p_{\text {int }}}+\mathbf{f}_{\sigma_{\text {int }}}=0$. Thus, the thrust of the jet is defined as:

$$
\mathbf{f}_{\mathrm{jet}} \equiv \mathbf{f}_{\Delta p_{\mathrm{ext}}}+\mathbf{f}_{\sigma_{\mathrm{ext}}}
$$

Finally, at equilibrium, we have:

$$
\mathbf{f}_{\mathrm{jet}}=-\mathbf{f}_{d}
$$

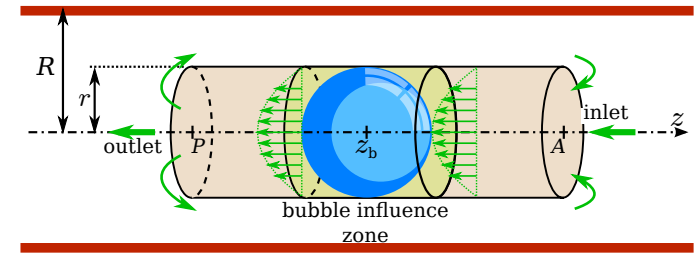

(a)

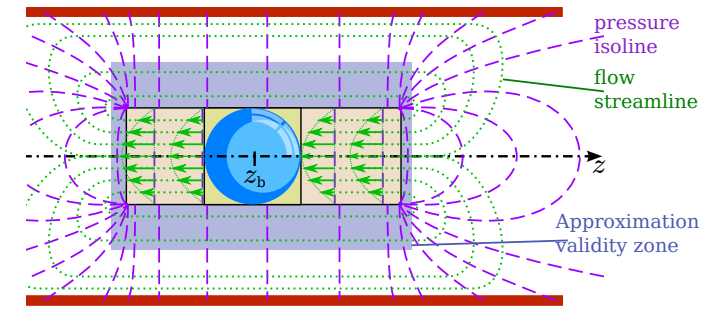

(b)

Fig. 5. Representation of (a) the microjet and the inner flow, and (b) pressure (dashed-lines) and flow streamlines (dotted-lines), under the simplifying approximations.

\section{Assumptions}

To simplify the analytical fluidic calculations, in the following the microjet is considered as a cylindrical microtube of radius $r$ and length $l$, as illustrated in Fig. 5a. The created bubble is spherical with a radius $r_{b}=r$. The blood vessel is assumed to be a cylindrical microchannel of radius $R$ with an infinite length. It is filled with a liquid (e.g. blood) considered to be with viscosity $\eta$. In the jet, the bubble generates an influence zone which is assumed to be cylindrical with length $2 r$. Outside of this bubble influence area, the microjet inner flow behaves as a Poiseuille flow and has a parabolic speed profile. At the level of the jet, the flow speeds are permanent, parallel to the $\mathbf{G z}$-axis and independent of $z$. The only nonpermanent variable is the pressure within the jet (see Fig. 5b).

Assuming that the microjet is on the centerline of the microchannel, the forces are coaxial with the jet. The flow rate has no retroactive effect on the bubble motion. The latter and its influence zone are perfect mechanical transmitters of the pressure forces to the jet. The contribution of the overpressure in the bubble is neglected, according to its sphericity. Similarly, the steric, the Brownian, the electrostatic and the Van der Waal's microforces are neglected [15].

The microjet propelling force $\mathbf{f}_{j e t}$ through the drag force $\mathbf{f}_{\mathrm{d}}$, given in (5), is first considered as being linked to jet velocity $v_{\text {jet }}$ by [7]:

$$
\mathbf{f}_{\mathrm{jet}}=\frac{2 \pi \eta l}{\ln (l / r)-0.72} v_{\mathrm{jet}}
$$

with $r=r_{\max }=r_{\min }$ the radius of the cylindrical microjet. An alternative calculation, not using this formula, is then carried out in a comparative perspective.

\section{Pressure Line}

The pressure along the $\mathbf{G z}$-axis, when the bubble is in the center of the jet, is shown in Fig. 6(a). It appears a discontinuity in the bubble influence area. Actually the 


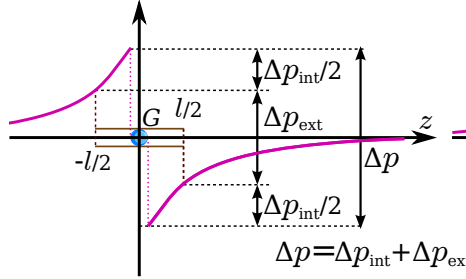

(a)

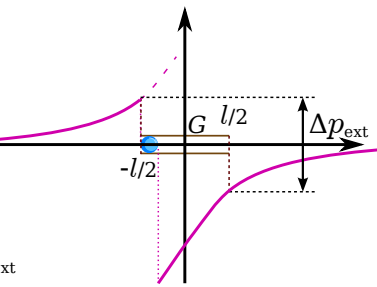

(b)
Fig. 6. Pressure line along the Gz-axis, when (a) the bubble is in the center of the jet, and (b) the bubble is positioned further to the rear of the jet (at the end of its migration).

pressure is not defined when $\forall z \in]-r ; r$. Let us notice that inside the jet, the pressure has a linear behavior. When the bubble moves back, the discontinuity shifts toward the rear $P$ of the jet, as depicted in Fig. 6(b). Finally, let us notice that the external pressure differential $\Delta p_{\text {ext }}$ remains constant when the bubble moves inside the microjet.

\section{PROPULSION OF THE MICROJET}

Considering the previous approximations, the microjet propulsion involves mainly the surrounding flow. Hence, the Navier-Stokes (N.-S.) equations together with the flow incompressibility allow the modeling of the microjet's engines. In this context, with the velocity vector expanded as $\mathbf{v}=\left(v_{x}, v_{z}\right)^{t}$, the N.-S. equations in cylindrical coordinates could be written as:

$$
\left\{\eta\left(\begin{array}{cc}
\partial_{z}^{2} v_{x}+\frac{1}{x} \partial_{x}\left(x \partial_{x} v_{x}\right)-\frac{v_{x}}{x^{2}} \\
\partial_{z}^{2} v_{z}+\frac{1}{x} \partial_{x}\left(x \partial_{x} v_{z}\right)
\end{array}\right)=\left(\begin{array}{c}
\partial_{x} p \\
\partial_{z} p
\end{array}\right)\right.
$$

with $x \in[0 ; R]$ the radial distance of the flow, and $z$ the component along the $\mathbf{G z}$-axis. The following is devoted to the study of the microjet's engines in its reference frame, first considering a static flow for the sake of simplicity.

\section{A. Engine Flow Outside of the Microjet}

In the backflow zone, the flow can be analytically calculated. Indeed, under the assumptions made in Section III, the N.-S. (7) system could be reduced as:

$$
\begin{aligned}
\exists \lambda>0 \\
{[\mathrm{~Pa} / \mathrm{m}] }
\end{aligned},\left\{\begin{aligned}
-\partial_{z} p & \equiv \lambda=\Delta p_{\text {ext, rect. }} / l \\
\partial_{x}\left(x \partial_{x} v_{z}\right) & =-\lambda \frac{x}{\eta} \\
\left(\partial_{x} p, \partial_{z}^{2} p\right)^{T} & \equiv(0,0)^{T}
\end{aligned}\right.
$$

Thus, there is a velocity field of the form $\mathbf{v}=v_{z}(x) \cdot \mathbf{e}_{z}$.

Let $\Lambda=R / r$ denote the vessel $(R) /$ microjet $(r)$ radii ratio. In the backflow zone, the motor flow speed $v_{z}(x)$ is expressed by solving (8) as follows:

$$
v_{z}(x)=\frac{\lambda r^{2}}{4 \eta}\left(\left(\Lambda^{2}-1\right) \frac{\ln (x / r)}{\ln \Lambda}+1-\left(\frac{x}{r}\right)^{2}\right)
$$

In the upstream and downstream of the jet, it is difficult to fully analytically characterize the driving flow. However, its velocity norm remains less than the bubble speed $v_{\mathrm{b}}$. In the backflow zone, let $v_{\max }$ denote the maximum motive speed, and $x_{\max } \in[r ; R]$ defined such as $v_{z}\left(x_{\max }\right)=v_{\max }$. Hence, a dimensional analysis shows that the component $\Delta p_{\text {ext,curv }}$ of $\Delta p_{\text {ext }}(3)$ is in the order of:

$$
\Delta p_{\text {ext }, \text { curv. }} \approx 2 \eta \frac{v_{\max }}{x_{\max }}
$$

The computation of the pressure gradient $\lambda=\Delta p_{\text {ext, rect. }} / l$ is performed by writing the flow conservation between the flow inside the jet and the backflow zone, which leads to:

$$
\lambda=\frac{8 \eta}{r^{2}} \frac{\ln (\Lambda) v_{\mathrm{b}}}{\left(\Lambda^{2}-1\right)\left(\left(\Lambda^{2}+1\right) \ln (\Lambda)+1-\Lambda^{2}\right)}
$$

\section{B. The Two Motors of the Microjet}

1) The pressure engine: The pressure force $\mathbf{f}_{\Delta p}$ exerted on a cylindrical microjet is bound to the pressure difference $\Delta p$ through the linear equation:

$$
\mathbf{f}_{\Delta p}=\pi r^{2} \Delta_{p}
$$

Similarly, the same goes for the pressure differential component given by (2) and (3). As mentioned, calculations show that the internal forces counteract each other. Furthermore, in small capillaries, the pressure differential (10) and the

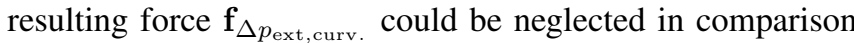
with the pressure differential $\Delta p_{\text {ext, rect. }}$ and its induced force

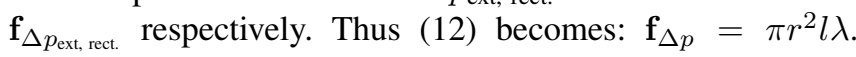
Hence, the pressure force is then:

$$
\mathbf{f}_{\Delta p_{\text {ext, rect. }}}=\frac{8 \pi \eta l v_{\mathrm{b}} \ln (\Lambda)}{\left(\Lambda^{2}-1\right)\left(\left(\Lambda^{2}+1\right) \ln (\Lambda)+1-\Lambda^{2}\right)}
$$

2) The backflow engine: The backflow driving force $\mathbf{f}_{\sigma_{\text {ext }}}$ is given by the integration of the stress $\sigma_{\text {ext }}$ related to the external wall of the catalytic microjet, and we get:

$$
\mathbf{f}_{\sigma_{\mathrm{ext}}}=\frac{4 \pi \eta l v_{\mathrm{b}}\left(\Lambda^{2}-1-2 \ln (\Lambda)\right)}{\left(\Lambda^{2}-1\right)\left(\left(\Lambda^{2}+1\right) \ln (\Lambda)+1-\Lambda^{2}\right)}
$$

3) The thrust of the microjet: Summing up the driving forces (13) and (14), we obtain the microjet propelling force:

$$
\mathbf{f}_{\mathrm{jet}}=\frac{4 \pi \eta l v_{\mathrm{b}}}{\left(\Lambda^{2}+1\right) \ln (\Lambda)+1-\Lambda^{2}}
$$

In arteries the shear force $\mathbf{f}_{\sigma_{\text {ext }}}$ is more significant than the pressure force $\mathbf{f}_{\Delta p_{\text {ext rect. }} \text {, but these two driving forces }}$ are both very weak, as depicted in Fig. 7. In capillaries these forces are maximum, and remain in the same order of magnitude. As the propelling forces are related to the flow viscosity, obviously the jet force is more significant in blood flow where classically the viscosity is in the range of $\eta \in[2.9 ; 6.5] \mathrm{mPas}$ in capillaries $(R \in[6 ; 60] \mu \mathrm{m})$, against aqueous solution with $\eta=1.13 \mathrm{mPa}$ s [7].

To compare the two microjet's engines, let us first examine their ratio:

$$
\frac{\mathbf{f}_{\sigma_{\text {ext }}}}{\mathbf{f}_{\Delta p_{\text {ext rect. }}}}=\frac{1}{2}\left(\frac{\Lambda^{2}-1}{\ln (\Lambda)}-2\right)
$$

This result exhibits a dependency only in $\Lambda$. The study of this function shows that in small vessel (e.g. in capillaries), these driving forces contribute equivalently. Finally, let us 


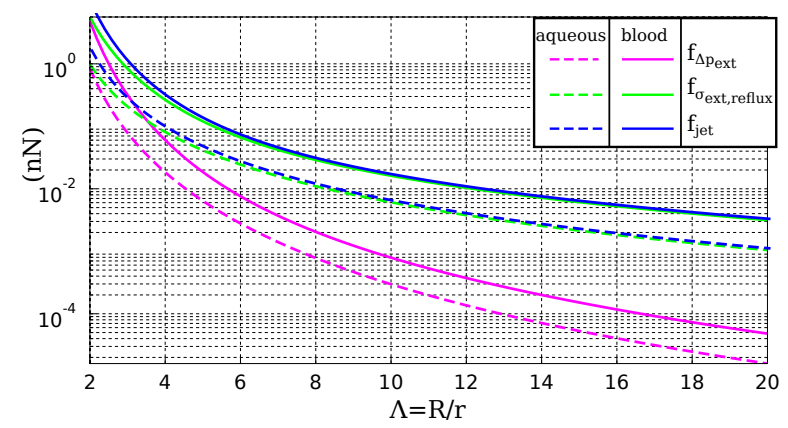

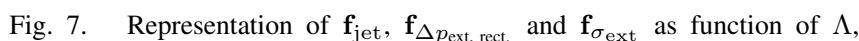
for $l=50 \mu \mathrm{m}$ and $v_{\mathrm{b}}=1.2 \mathrm{~mm} / \mathrm{s}$ in blood $(\eta \in[2.9 ; 6.5] \mathrm{mPas})$ and aqueous solution $(\eta=1.13 \mathrm{mPas}[7])$ in capillaries.

define the ratio that exhibits the relative contribution of the pressure force, that is:

$$
\tau=\frac{\mathbf{f}_{\Delta p_{\text {ext, rect. }}}}{\mathbf{f}_{\text {jet }}}=\frac{2 \ln (\Lambda)}{\Lambda^{2}-1}
$$

Fig. 8 shows the evolution of the pressure force contribution as function of the ratio $\Lambda$. In large vessel, where $\Lambda \gg 1$, the pressure force is less efficient.

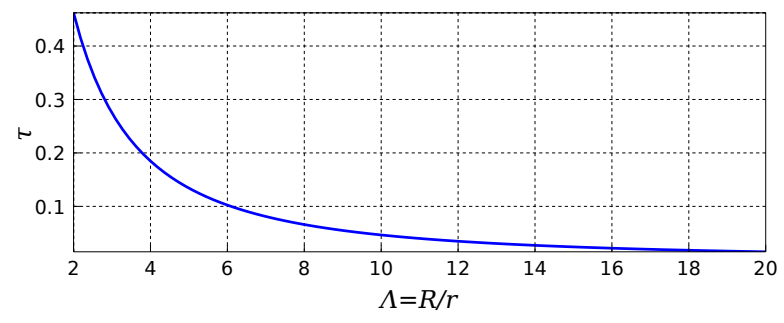

Fig. 8. The relative contribution of the pressure force.

C. The link between the velocities of the bubble and the microjet

1) Applying the standard drag formula: Combining Eq. (6) and (15), the relationship (1) can be specified as:

$$
v_{\text {jet }}=\frac{2(\ln (l / r)-0.72)}{\left(1+\Lambda^{2}\right) \ln (\Lambda)+1-\Lambda^{2}} \cdot v_{\mathrm{b}}
$$

As one can see, it appears that $v_{\text {jet }}$ is independent of the liquid's viscosity $\eta$, and is mainly related to the geometry of the jet and the vessel's size. Thus, the jet presents some scale invariance. Furthermore, the smaller the ratio $\Lambda$ is, the higher is the velocity of the microjet.

For instance, with $\Lambda=2$ and $l / r=50 / 3$ the microjet velocity is about $v_{\text {jet }} \approx 9 v_{\mathrm{b}}$. Considering a bubble moving at $v_{\mathrm{b}}=1.2 \mathrm{~mm} / \mathrm{s}$ the jet speed is about $v_{\text {jet }} \approx 10.8 \mathrm{~mm} / \mathrm{s}$, as illustrated in Fig. 9 with the red dashed-line. Compared to the literature [6]-[10], [13], these results are about ten times higher. The explanation for this discrepancy is due to two main reasons. First, the considered environments are different. In previous work, the authors have conducted their experiments mainly in water and infinite extent (i.e. without microjet/wall influence). Secondly, the jet propelling force formula (6) does not take into account the geometrical

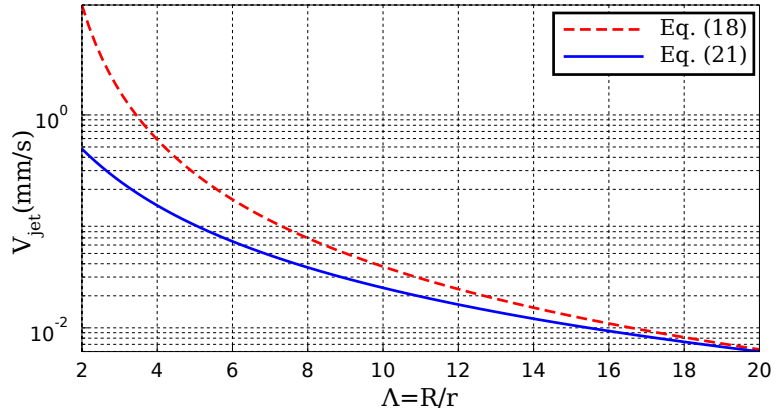

Fig. 9. Velocity of the microjet $v_{\text {jet }}$ as function of $\Lambda$, with $l / r=50 / 3$ and the bubble's velocity $v_{\mathrm{b}}=1.2 \mathrm{~mm} / \mathrm{s}$, computed from (18) and (21).

characteristics of its environment. Therefore is not always appropriate, especially in thin vessels.

2) Using drag force with the Navier-Stokes equations: To overcome the weakness of the above modeling, we propose to replace the standard jet propelling force formula (6) with one derived from the analytical treatment of the N.-S. equations. To compute the drag influence, the flow estimation in the backflow zone is approximately carried out. Hence, similar calculations to those relating to the engine flow are realized. The drag force $f_{d}$ is considered as the sum of a shear and pressure forces, that is:

$$
\mathbf{f}_{\mathrm{d}}=\mathbf{f}_{\mathrm{d} \sigma}+\mathbf{f}_{\mathrm{d} \Delta p}
$$

Furthermore, the drag pressure is assumed constant over the whole section of each orifice of the jet, and equal to the nearest shear pressure in the backflow zone. After all computations, the following drag force is obtained:

$$
\mathbf{f}_{\mathrm{d}}=-\frac{2 \pi \eta l\left(\Lambda^{2}+1\right)}{\left(\Lambda^{2}+1\right) \ln (\Lambda)+1-\Lambda^{2}} v_{\text {jet }}
$$

Using (20) with (15), it is straightforward to obtain a simple formula that relates $v_{\text {jet }}$ and $v_{\mathrm{b}}$, and the proposed relationship (1) becomes:

$$
v_{\text {jet }}=\frac{2 v_{\mathrm{b}}}{\Lambda^{2}+1}
$$

Hence, for a bubble motion at $v_{\mathrm{b}}=1.2 \mathrm{~mm} / \mathrm{s}$ the microjet velocity remains $v_{\text {jet }} \leq 1.2 \mathrm{~mm} / \mathrm{s}$, as represented in Fig. 9 with the blue plain-line. This result is consistent with previous studies [6]-[10], [13]. Particularly, a maximal jet velocity of about $v_{\text {jet }} \leq 1500 \mu \mathrm{m} / \mathrm{s}$ has been reported [8], [10]. On the other hand, the impact of the drag pressure is more significant in capillaries, but is negligible in larger vessels. Nevertheless, it allows bounding the jet speed. Finally, contrary to (18), in Eq. (21) $v_{\text {jet }}$ depends mainly on the ratio $\Lambda$. In this case, the microjet aspect ratio $l / r$ becomes less relevant. This also exhibits a scale invariance in the microjet motion.

\section{The Microjet's Geometry, Forces and Velocities Analysis}

Fig. 10 represents the evolution of the ratios $\left(\tau, v_{\text {jet }} / v_{\mathrm{b}}\right)$ for different geometry factors $\Lambda$ (see also Fig. 8). Thus, it links the relative contribution of the pressure force $\mathbf{f}_{\Delta p_{\text {ext, rect. }}}$ 
to the microjet velocities. Both ratios are varying between 0 to 1 . This curve clearly shows that they are positively correlated. Secondly for $\Lambda \leq \Lambda_{0}$ (with $\Lambda_{0}=1.874$ ), the pressure force is the main driving force of the microjet. In this study, $\Lambda \geq 2$ has been mainly focused with respect to the considered approximations (blue plain line in Fig. 10). In particular, these approximations consider the available manufactured microjets conical angles. For smaller values of $\Lambda$ (red dashed line in Fig. 10), lower conical angles have to be designed. Thus, these microjets would be the most efficient ones. Actually the microjet's propulsion would mainly choose the most efficient way: the pressure way.

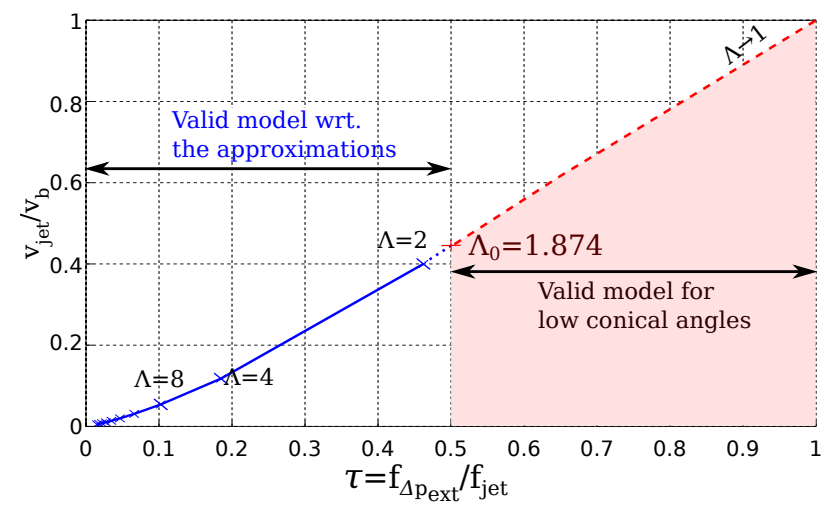

Fig. 10. Curve of the ratios $\left(\tau, v_{\text {jet }} / v_{\mathrm{b}}\right)$ for different geometry $\Lambda$. The speed ratio $v_{\text {jet }} / v_{\mathrm{b}}$ is an increasing function of the pressure force contribution, which is maximum for the weakest values of $\Lambda$.

\section{DISCUSSIONS}

The proposed microjet propulsion model is simple under the considered approximations. The associated calculations are conducted thanks to approximations of the N.-S. equations, which can be gathered in three groups: i) geometrical and mechanical aspects that concern mainly the walls of the microjet and the vessel, and influence the force approximations; ii) flow with its behavior around the jet; and iii) forces approximations, which involve their orientations and the link between the drag force and the velocity of the microjet. Furthermore, the obtained results had to be in good agreement with the literature. For instance Eq.(21) matches suitably the numerical and experimental results presented in [6]-[10], [13].

Some hypothesis could be further investigated, such as the bubble's sphericity, or its mechanical contact with the jet wall and its growing speed, as suggested in [7]. The geometry flaw of the microjet has to be considered in relation to the study realized in [8]. Especially, in [11], [16] the authors have shown that the microjet has a natural deflection as nanorods. This mechanism makes microjet propulsion very interesting since, up to now, microtubular jets have generated higher propulsion forces compared to nanorods, spherical Janus particles, or microhelices [7], [10].

\section{CONCLUSION}

Catalytic tubular microjets are a promising candidates for innovative biomedical applications. They are thin, fast, powerful in small vessels, self-propelled, cheap to produce, weakly deflected and easily remote-controllable. Hence, they seem to be the ideal candidate to go into capillary and carry drugs to specified cells, long ahead other microsystems, such as controlled microorganism (e.g. magnetotactic bacteria). Their functioning remains not fully understood but the rough calculus performed in this article suggest how efficient these microswimmers are. This new understanding, in good agreement with the literature, needs to be further investigated to get closer to their real behavior. Some numerical simulations shall be performed to precise flow calculations. Especially, the exact contribution of the curved part of the flow streamlines has to studied.

\section{REFERENCES}

[1] A. A. Solovev, W. Xi, D. H. Gracias, S. M. Harazim, C. Deneke, S. Sanchez, and O. G. Schmidt, "Self-propelled nanotools," ACS Nano, vol. 6, no. 2, pp. 1751-1756, 2012.

[2] J. Wang and W. Gao, "Nano/microscale motors: biomedical opportunities and challenges," ACS nano, vol. 6, no. 7, pp. 5745-5751, 2012.

[3] W. F. Paxton, S. Sundararajan, T. E. Mallouk, and A. Sen, "Chemical locomotion," Angew. Chem. Int. Edit., vol. 45, no. 33, pp. 5420-5429, 2006.

[4] W. Xi, A. A. Solovev, A. N. Ananth, D. H. Gracias, S. Sanchez, and O. G. Schmidt, "Rolled-up magnetic microdrillers: towards remotely controlled minimally invasive surgery," Nanoscale, vol. 5, no. 4, pp. 1294-1297, 2013

[5] Y. Mei, A. A. Solovev, S. Sanchez, and O. G. Schmidt, "Rolled-up nanotech on polymers: from basic perception to self-propelled catalytic microengines," Chem. Soc. Rev., vol. 40, no. 5, pp. 2109-2119, 2011.

[6] G. Zhao, S. Sanchez, O. G. Schmidt, and M. Pumera, "Poisoning of bubble propelled catalytic micromotors: the chemical environment matters," Nanoscale, vol. 5, no. 7, pp. 2909-2914, 2013.

[7] V. M. Fomin, M. Hippler, V. Magdanz, L. Soler, S. Sanchez, and O. G. Schmidt, "Propulsion mechanism of catalytic microjet engines," IEEE Trans. Robot., vol. 30, no. 1, pp. 40-48, 2014.

[8] W. Gao, S. Sattayasamitsathit, J. Orozco, and J. Wang, "Highly efficient catalytic microengines: template electrosynthesis of polyaniline/platinum microtubes," J. of ACS, vol. 133, no. 31, pp. $11862-$ $11864,2011$.

[9] I. S. Khalil, V. Magdanz, S. Sanchez, O. G. Schmidt, and S. Misra, "The control of self-propelled microjets inside a microchannel with time-varying flow rates," IEEE Trans. Robot., vol. 30, no. 1, pp. 49$58,2014$.

[10] J. Li, G. Huang, M. Ye, M. Li, R. Liu, and Y. Mei, "Dynamics of catalytic tubular microjet engines: dependence on geometry and chemical environment," Nanoscale, vol. 3, no. 12, pp. 5083-5089, 2011.

[11] W. F. Paxton, K. C. Kistler, C. C. Olmeda, A. Sen, S. K. St. Angelo, Y. Cao, T. E. Mallouk, P. E. Lammert, and V. H. Crespi, "Catalytic nanomotors: autonomous movement of striped nanorods," $J$. of the American Chemical Society, vol. 126, no. 41, pp. 13 424-13 431, 2004

[12] B. J. Nelson, I. K. Kaliakatsos, and J. J. Abbott, "Microrobots for minimally invasive medicine," Annual Review of Bio.med. Eng., vol. 12, no. 1, pp. 55-85, 2010.

[13] S. Balasubramanian, D. Kagan, C.-M. Jack Hu, S. Campuzano, M. J. Lobo-Casta

;non, N. Lim, D. Y. Kang, M. Zimmerman, L. Zhang, and J. Wang, "Micromachine-enabled capture and isolation of cancer cells in complex media," Angew. Chem. Int. Edit., vol. 50, no. 18, pp. 4161-4164, 2011

[14] J. J. Abbott, M. C. Lagomarsino, L. Zhang, L. Dong, and B. J. Nelson, "How should microrobots swim?" The Int. J. of Robot. Res., 2009.

[15] K. Belharet, D. Folio, and A. Ferreira, "Simulation and planning of a magnetically actuated microrobot navigating in the arteries," IEEE Trans. Biomed. Eng., vol. 60, no. 4, pp. 994-1001, 2013.

[16] L. Baraban, S. M. Harazim, S. Sanchez, and O. G. Schmidt, "Chemotactic behavior of catalytic motors in microfluidic channels," Angew. Chem., vol. 125, no. 21, pp. 5662-5666, 2013. 